\title{
Electroreductive Methylation of Primary and Secondary Amines
}

\author{
Renato A. da Silva*, Ronny F. M. de Souza, Madalena C. C. Areias, Marcelo Navarro \\ and Lothar W. Bieber
}

Departamento de Química Fundamental, CCEN, Universidade Federal de Pernambuco

*renatoagtsilva@gmail.com

Keywords: electroreductive amination, iminium ions and formaldehyde.

\section{INTRODUCTION}

A new synthetic method of reductive methylation promoted by electrolysis in aqueous medium was studied in this work. The method is based on the reduction of iminium ions generated in situ from the condensation of amines with formaldehyde. Besides the classical Leuckart-Wallach or Eschweiler-Clarke procedures, ${ }^{1}$ this reaction is normally performed in organic solvents using several borohydride reagents ${ }^{2}$ or catalytic hydrogenation. ${ }^{3}$ We decided to study the electrorreductive methylation reaction, by means of aqueous formaldehyde motivated by the good results of our reaction promoted by zinc ${ }^{4}$ and due to the scarce reports on the electrochemical strategy in the literature. 5,6

\section{RESULTS AND DISCUSSION}

Our experiments showed that $N$-methylated amines are prepared in good yields by potential-controlled reduction of aqueous formaldehyde at a carbon cathode in acid medium containing primary or secondary amines. Table 1 shows the results for methylation of morpholine, piperidine and diethylamine as well as the dimethylation of benzylamine. The product of monoalkylation with benzylamine was not detected by GC.

Table 1. Electroreductive methylation. ${ }^{\text {a }}$

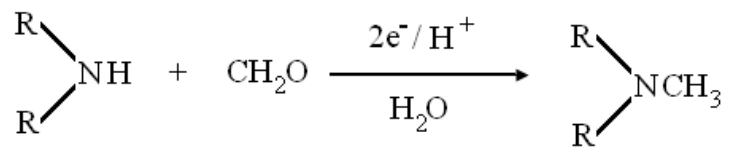

\begin{tabular}{|l|l|l|}
\hline Amines & Potential (V) & Yield (\%) \\
\hline Morpholine & $-1,6$ & 80 \\
Piperidine & $-1,7$ & 70 \\
$N, N$-dietylamine & $-1,6$ & 92 \\
Benzylamine ${ }^{b}$ & $-1,6$ & 60 \\
\hline
\end{tabular}

${ }^{\text {Reaction }}$ conditions: amine $(5 \mathrm{mmol})$, formaldehyde
acetic acid (20mmol). Time : 4 hours. ${ }^{b}$ Dimethylation.
Figure 1 show a schematic representation of the electrochemical cell containing carbon graphite (cathode and anode) and saturated calomel electrode (reference) used in this work.

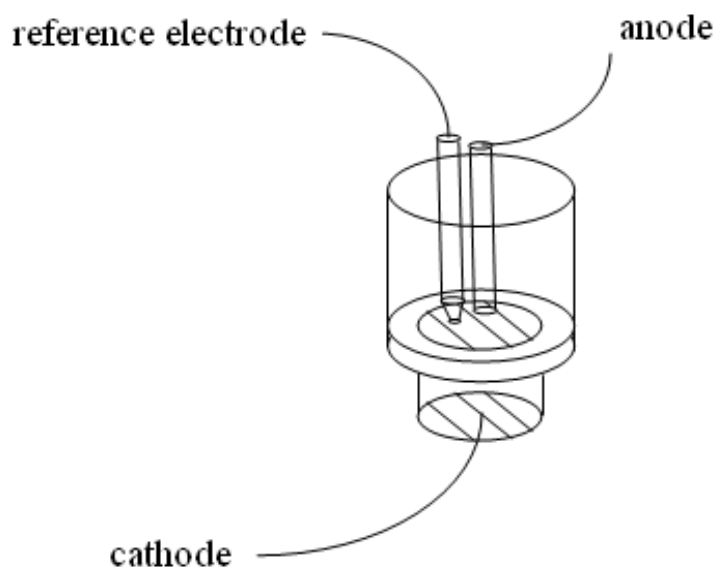

Figure 1. Electrochemical cell

\section{CONCLUSION}

In summary, electrorredutive amination may be considered as a good alternative for the preparation of $\mathrm{N}$-methylamines in good yield. The present methodology is simple, safe, of low toxicity and reproducible, can be applied at ambient temperature without the necessity of use of an inert atmosphere.

\section{ACKNOWLEDGEMENTS}

This work was supported by fellowships and grants from CNPq (Brasilia). \footnotetext{
REFERENCES

${ }^{1}$ Moore, M. L. Organic Reactions 1949, 5, 301-303.

${ }^{2}$ Baxter, E. W.; Reitz, A. B. Organic Reactions 2002, 59, 1-714.

${ }^{3}$ Emerson, W. S. Organic Reactions 1948, 4, 174-255.

${ }^{4}$ Silva, R. A.; Bieber, L. W.; Estevam, I. H. S. Tetrahedron Letters 2007, 48, 7680-7682.

${ }^{5}$ Lund, H. Acta Chem. Scand. 1959, 13, 249.

${ }^{6}$ Smirnov, Y.; Fedorova, L. A.; Tomilov, A. P. Soviet electrochemistry 1992 28 (4), 476-484.
} 